\title{
Congenital myopathies : characteristics and subtypes in Hong Kong
}

Chan Sophelia HS'1, Ho Ronnie SL², Chan Angel OK², Ip Janice JK33, Wong Shun4, Ng Grace SF5, Lee Hencher $\mathrm{CH}^{6}$, Cheng Yue $^{7}$, Liu KT ${ }^{8}$, Lee CN9 , Fung Sharon $\mathrm{TH}^{10}$, Cherk Sharon WW ${ }^{10}$, Chan Timothy SK ${ }^{11}$, Lam Wendy MW³, Shek WH², Wong Virginia $\mathrm{CN}^{1}$

${ }^{1}$ Department of Paediatrics and Adolescent Medicine, Queen Mary Hospital, The University of Hong Kong; ${ }^{2}$ Department of Pathology and Clinical Biochemistry \& 3 Department of Radiology ,Queen Mary Hospital, HKSAR; ${ }^{4}$ Department of Pathology , 5 Department of Paediatrics and Adolescent Medicine \& ${ }^{6}$ Department of Clinical Pathology, Princess Margaret Hospital, HKSAR; 7 Department of Pathology, ${ }^{8}$ Department of Paediatrics and Adolescent Medicine, \& 9 Department of Medicine and Geriatrics, Pamela Youde Nethersole Eastern Hospital, HKSAR; ${ }^{\circ}$ Department of Paediatrics \& "Department of Pathology, Kwong Wah Hospital, HKSAR

Background: Congenital myopathies (CMs) are a genetically and clinically heterogeneous group of neuromuscular disorders. Historically, the congenital myopathies are classified according to muscle biopsy findings - Rods (Nemaline myopathy) (NM), cores (central core disease and multiminicore disease) (Core and MMC), central nuclei (centronuclear/ myotubular myopathy)(CNM), and selective hypotrophy of type 1 fibres ( congenital fibre type disproportion CFD). Over twenty genes have been implicated in CMs. The overlapping clinical presentations among different histopathological findings and different mutations poses major diagnostic challenge.

Objective: We investigated the characteristics of children with congenital myopathies in Hong Kong.

Patients and methods: We identified all patients with a confirmed diagnosis of CM between 2012-March 2015. Their clinical presentation , muscle biopsy, muscle MRI and genetic analysis results were evaluated.

\section{Results:}

Patients:

Total 15 patients have been diagnosed to have CM. Nine were males (6o\%), 6 were female (40\%).

\section{Genetic findings:}

A genetic diagnosis could be established in $11(73 \%)$ out of 15 patients. Among those 11 patients, 4 (36\%) were mutated in $R Y R{ }_{1}, 3(27 \%)$ in $A C T A 1$ $2(18 \%)$ in $K L H L 40,1(9 \%)$ in $M T M 1$ and $1(9 \%)$ in DNM2. A total of 13 mutation were identified

(2) The missense RYRi mutation (c.3523G>A) was found in 2 patients, and the missense KLHL4O mutation (c.1516A>C) was found in another 2 patients, suggesting that these variants could probably be the hot spots mutation among Chinese patients.

(3) Pathological heterogeneity caused by $R Y R ı$ mutation is shown in our 4 patients showing different findings including nemaline rods, central cores, multiminicores, or type 1 fibre predominance.

Histopathological features:

(1) Muscle biopsy evaluation were available in all 15 patients. Nemaline myopathy were the most frequent histopathological diagnosis, in 5 patients $(33 \%)$, followed by core myopathy, in 4 patients $(26 \%)$, centronuclear myopathy in 2 patients (13\%), congenital fibre type disproportion in 2 patients (13\%), zebra bodies in $1(6.7 \%)$ patient and type 1 predominance in $1(6.7 \%)$ patient.

(2) Genetic heterogeneity is illustrated in our patients with nemaline myopathy. Amongst the 5 patients, 1 had RYR1, 2 had ACTA 1 and 2 had KLHL40 mutation.

Clinical features:

(1) Of the 15 patients, $9(60 \%)$ had age of onset at birth or before one month, $3(20 \%)$ between 1 and 12 months, and $3(20 \%)$ between 1 and 5 years. Out of the 9 patients with early neonatal presentation, $3 / 9$ (33\%) patients died before 13 months.

(2) The functional abilities varied from very severe weakness required tube feeding and ventilation support , to intermediate functional abilities with possible independent sitting, to mild limb girdle weakness only.

(3) $A C T A 1, K L H L 40, D N M 2$ and $M T M 1$ mutations are associated with severe presentation with early neonatal onset.

(4) RYRı mutations are associated with a milder phenotype with all the affected patients maintain independent walking

Muscle imaging:

Selective muscle involvement with Rectus Femoris sparing provides helpful clues to a possible underlying RYRı mutation.

\section{References:}

I Colombo, M Scoto, AY Manzur et al. Congenital myopathies Natural history of a large pediatric cohort. Neurology 2015;84:28-35 KN North, CH Wang, N Clarke, et al. Approach to the diagnosis of congenital myopathies. Neuromuscular Disorders 2014:24: 97-116 L Maggi, M Scoto, S Cirak, et al. Congenital myopathies- clinical features and frequency of individual subtypes diagnosed over a 5 year period in the United Kingdom. Neuromuscular Disorders 2013:23:195-205

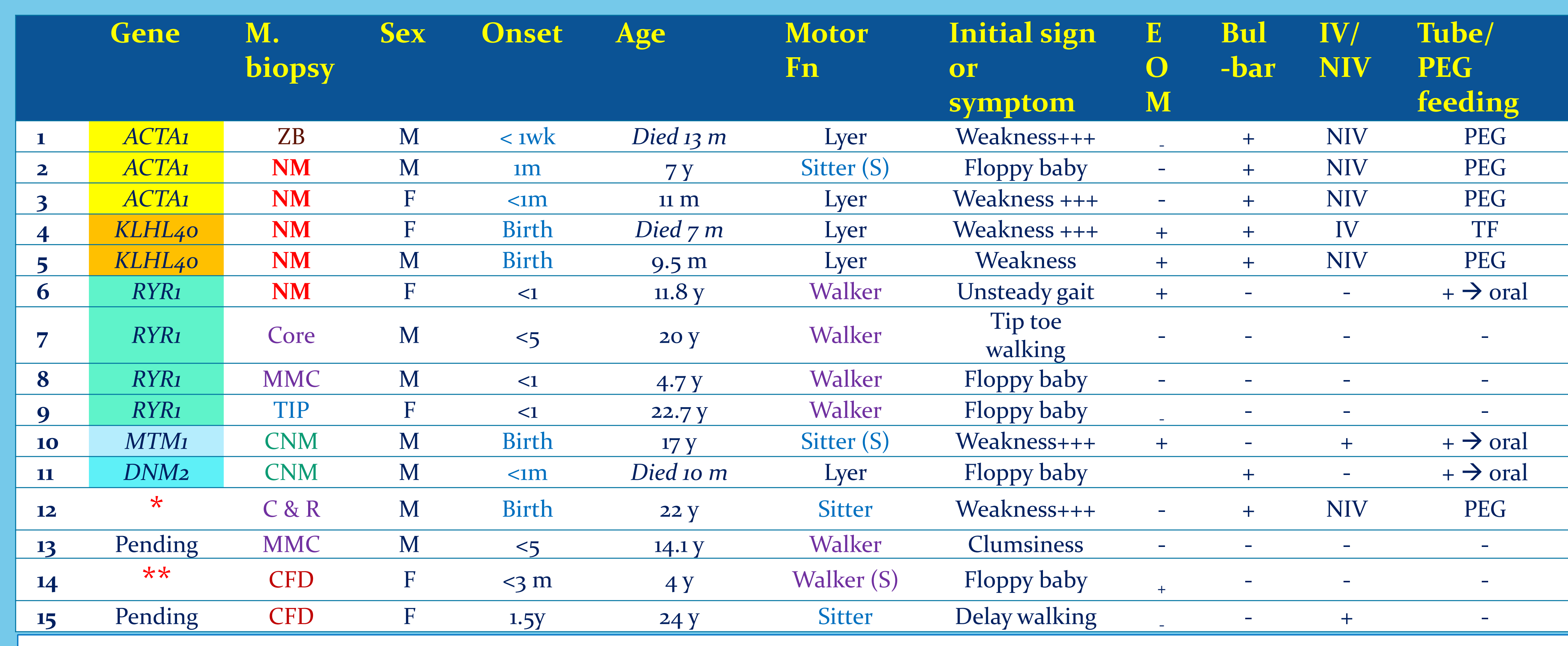

\begin{tabular}{|c|c|c|c|c|c|}
\hline & Gene & $\begin{array}{l}\text { Muscle } \\
\text { biopsy }\end{array}$ & Mutation & $\begin{array}{l}\text { Inheritance } \\
\text { Pattern }\end{array}$ & $\begin{array}{c}\text { Parents' } \\
\text { carrier status }\end{array}$ \\
\hline 1 & ACTA1 & ZB & c.529A>G (p.Ile177Val) & $A D$ & No \\
\hline 2 & ACTA1 & NM & c.80zT>C (p.Phez68Leu) & AD & No \\
\hline 3 & ACTA1 & NM & c.547G>A (p.Ala183Thr) & $A D$ & No \\
\hline 4 & KLHL4O & NM & c.1516A>C(p.Thr5o6Pro) & AR & Yes \\
\hline 5 & KLHL4O & NM & c.1327G>A(p.Gly443Ser) + c.1516A>C(p.Thr5o6Pro) & $A R$ & Yes \\
\hline 6 & $R Y R 1$ & NM & c.380oC>G (p.Pro1267Arg) + c.1675dup (p.11e559Asnfs* $\left.{ }^{*} 11\right)$ & $A D$ & Yes \\
\hline 7 & $R Y R_{1}$ & Core & c. $7523 \mathrm{G}>\mathrm{A}$ (p.Arg2508His) & $A R$ & No \\
\hline 8 & $R Y R_{1}$ & MMC & $\begin{array}{l}\text { c.3523G >A (p.Glu1175Lys) + c.11956dupG } \\
\text { (p.Asp3986Glyfs*89) }\end{array}$ & $A R$ & Yes \\
\hline 9 & $R Y R{ }_{1}$ & TIP & $\begin{array}{l}\text { c.3523G>A (p.Glu1175Lys) + c.10615delC } \\
\text { (p.Arg3539Valfs* } 4 \text { ) }\end{array}$ & AR & Yes \\
\hline 10 & MTMi & CNM & c.1644+2T>C (p?) a splicing mutation & X-linked & No \\
\hline 11 & $\mathrm{DNM}_{2}$ & CNM & c.1124T>A (p. Val375Glu) & $A D$ & No \\
\hline
\end{tabular}

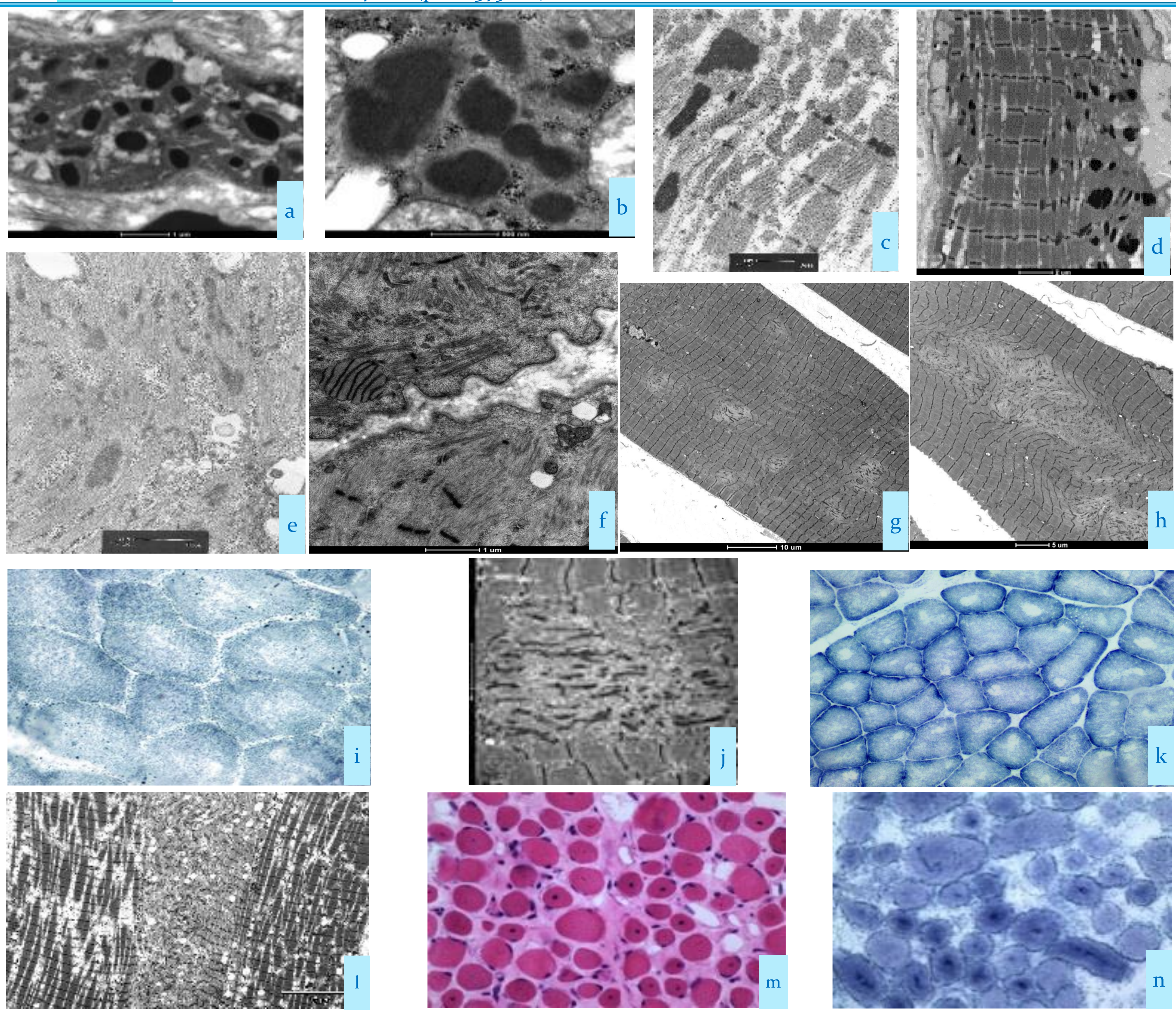

(a \&b). Electron microscopy (EM) of muscle biopsies of patients 4 \& 5 with nemaline myopathy due to KLHL4O mutation with roundish rods; (c\&d). Electron microscopy of muscle biopsies of patient 2 \& 3 with nemaline myopathy due to ACTA1 mutation; (e) Muscle biopsy of patient 12 with central core disease having rods shown on EM; (f) A zebra body is noted on the EM of patient 1 with $A C T A 1$-related congenital myopathy when the muscle biopsy was performed at 1.5 month old; (g \& h) Muscle biopsy of patient 13 with multi-minicores on the EM; (i \& j) Muscle biopsy of patient 8 with multi-minicore disease due to $R Y R 1$ mutation showing uneven staining with SDH in some fibres and EM shows a large minicore with excess Z-line material and myofilament disruption; ( $\mathrm{k} \& \mathrm{l}$ ) Muscle biopsy of patient 7 with central core myopathy due to $R Y R_{1}$ mutation with NADH shows numerous cores and EM shows a central core in the centre with disrupted Z-line. ( $m \& n$ ) Muscle biopsy of patient 11 with centronuclear myopathy due to DNM2 mutation. Central nuclei are seen in some fibres (H\&E) and no radiating strands are noted from the central nuclei (NADH). 